Archives

$27 \mid 2001$

Officiers "moyens" (II)

\title{
Les frontières brouillées du privilège
}

Officiers royaux et seigneuriaux dans l'apanage d'Orléans aux XVII ${ }^{\mathrm{e}}$ et $\mathrm{XVII}^{\mathrm{e}}$ siècles

\section{Katia Béguin}

\section{OpenEdition Journals}

\section{Édition électronique}

URL : http://journals.openedition.org/ccrh/1273

DOI : $10.4000 /$ ccrh. 1273

ISSN : 1760-7906

\section{Éditeur}

Centre de recherches historiques - EHESS

Édition imprimée

Date de publication : 20 octobre 2001

ISSN : 0990-9141

\section{Référence électronique}

Katia Béguin, «Les frontières brouillées du privilège », Les Cahiers du Centre de Recherches Historiques [En ligne], 27 | 2001, mis en ligne le 23 novembre 2008, consulté le 01 mai 2019. URL : http:// journals.openedition.org/ccrh/1273; DOI : 10.4000/ccrh.1273

Ce document a été généré automatiquement le 1 mai 2019. 


\title{
Les frontières brouillées du privilège
}

\author{
Officiers royaux et seigneuriaux dans l'apanage d'Orléans aux XVII ${ }^{\mathrm{e}}$ et \\ $\mathrm{XVII}^{\mathrm{e}}$ siècles
}

\section{Katia Béguin}

1 Cette contribution à la connaissance du milieu des officiers royaux et seigneuriaux est le fruit d'une découverte fortuite et heureuse de documents d'une grande richesse : il s'agit de brouillons de mémoires et de traités composés au milieu du XVIII ${ }^{e}$ siècle, sans doute à la demande des ducs d'Orléans, par un ou plusieurs juristes à leur service. Ces manuscrits forment pour certains la matière d'ouvrages destinés à être publiés : une partie au moins l'a sans doute été ; mais ces premiers jets et les rectifications ultérieures s'avèrent plus intéressants que l'éventuelle version imprimée, parce qu'ils comportent des passages biffés mais lisibles, qui montrent la manière dont on a puisé, éliminé, sélectionné des arguments au sein d'un arsenal plus vaste de justifications pour la défense des droits de l'apanage des ducs d'Orléans. En outre, ces sources livrent des réflexions sur la vénalité et ses effets, dévoilent la manière dont ses évolutions conditionnent les pratiques d'une aristocratie attentive aussi bien aux rentrées d'argent qui alimentent ses parties casuelles qu'aux prérogatives et aux honneurs attachés au pouvoir d'instituer et même de nommer au roi des officiers. C'est en cela aussi que ces papiers s'avèrent précieux, si l'on songe que David Bien regrettait que l'historien des offices soit souvent contraint de déduire les modes de raisonnement, sinon les «stratégies » des acteurs, des documents témoignant des pratiques, lesquels l'emportent de loin sur le commentaire parmi les sources ${ }^{1}$. Au contraire, ces deux aspects se trouvent associés en permanence dans les documents en question. Aussi y avait-il là une fenêtre monographique intéressante, faute d'être représentative, en raison des spécificités très fortes qui découlent du rang éminent des ducs d'Orléans dans la monarchie aux XVII et XVIII ${ }^{\mathrm{e}}$ siècles, successivement frère du roi, fils de France, puis premiers princes du sang, et tous princes apanagés depuis 1626. Elle paraît néanmoins indicative des choix opérés, des motivations, du comportement des grands et de leurs serviteurs face aux mutations de la politique royale de l'office. Une voie 
transversale pour aborder le problème de la crise des offices au XVIII ${ }^{\mathrm{e}}$ siècle s'offre ainsi, dans le sillon tracé par des travaux récents, qui se refusent à traiter les vicissitudes officières comme une donnée universelle, uniforme ou inéluctable, pour les réinscrire dans leur contexte spécifique et éclairer leurs variantes géographiques ou chronologiques 2 . Elle offre la matière d'une réflexion à l'envers sur les raisons de la désaffection vis-à-vis de la «marchandise d'État », lorsqu'elle est avérée. Je m'attacherai à démêler la finalité de ces recherches juridiques, qui découvrent un univers privilégié de l'office moyen, royal ou seigneurial, dont la singularité se trouve cependant menacée au milieu du xviiie siècle.

\section{Un inventaire opportun des droits et des offices}

2 Il paraît légitime de réfléchir aux conditions de l'apparition presque simultanée, chez les Condé et les Orléans, de ce qui constitue pour nous un matériau historique nouveau. Dès le milieu du xVIII ${ }^{\mathrm{e}}$ siècle, la même nécessité se fait jour, au moins pour ces deux grandes maisons aristocratiques, d'établir un état des offices, des revenus casuels et des droits respectifs des princes à nommer ou à instituer des officiers. Cette démarche, qui suscite de part et d'autre une production de documents similaires, mais plus explicites pour les Orléans (les seuls exploités ici), ne peut être ramenée au simple volet particulier d'une plus large entreprise de valorisation économique des biens fonciers et domaniaux de la haute noblesse. Ce serait réducteur, semble t-il, d'autant que la destination et la nature des documents, imprimés ou non, indiquent qu'ils s'inscrivent avant tout dans une optique défensive, imposée par des circonstances nouvelles. Bien entendu, ces inventaires débutent alors que l'œuvre de rationalisation menée à l'instigation du bureau de Législation du chancelier D'Aguesseau commence à produire ses effets, avec les édits particuliers qui commandent des fusions de tribunaux ou la suppression des châtellenies et vigueries dans les villes sièges de bailliages ou de sénéchaussées, imposée par l'édit général de $1749^{3}$. De toute évidence, le dénombrement des offices effectué pour les Orléans prend acte de ces remaniements de la carte judiciaire. Mais le rédacteur révèle de manière indirecte, à l'occasion d'une mention des officiers comptables, l'une des raisons de l'énumération entreprise : l'injonction vient pour une part du contrôle général des finances, qui réclame au chancelier du duc un état des offices de finances sans titulaires afin d'examiner le problème suscité par l'augmentation de la finance et des gages qui leur est imposée par un édit de 1743, et dont les Orléans se trouvent de fait redevables ${ }^{4}$. Les dates portées dans chacune des parties de l'ouvrage témoignent, à tout le moins, de la durée de cette compilation méthodique des édits de création, des règlements ultérieurs, des arrêts du Conseil regardant la maison d'Orléans, qui excède de loin la demande ponctuelle de Machault d'Arnouville : la première partie, composée en 1747, n'est suivie d'une seconde que trois années plus tard ${ }^{5}$. L'auteur souligne d'ailleurs combien son travail exige

[...] la circonspection la plus exacte, et pour la régie des revenus casuels de Monseigneur le duc d'Orléans, et pour l'expédition des lettres qui émanent de sa chancellerie, afin d'établir à cet égard un ordre inviolable ${ }^{6}$.

D'autres remarques insérées en maint endroits de l'inventaire découvrent que la conjonction de plusieurs facteurs ébranle alors la solidité des prérogatives ducales. Deux procès, d'apparence banale, intentés aux Orléans par des officiers, l'un pendant la décennie 1720, l'autre en 1746, paraissent bien être - le second surtout - le motif direct de la rédaction, commencée peu après, du traité qui comporte en effet un récit circonstancié 
de la bataille judiciaire encore ouverte en $1752^{7}$. La contestation s'est introduite à la faveur des multiples changements apportés au statut des offices royaux durant le siècle et demi qui a suivi l'instauration de la vénalité légale, et en particulier de deux pratiques répandues durant les dernières décennies du règne de Louis XIV : la création d'offices héréditaires ou l'attribution de l'hérédité à une partie d'entre eux, moyennant une taxe équivalente à un emprunt forcé. À cet égard, le second procès a révélé une faille béante du droit ducal et les conséquences redoutables qu'elle laissait augurer :

[...] c'est, on ose l'avouer, la plus intéressante de toutes celles [les questions] qui peuvent concerner la maison d'Orléans, et il n'y a nul danger d'exagérer en disant que, dans le cas où ses adversaires obtiendraient gain des causes, il deviendrait inévitable à l'égard de Mgr le duc d'Orléans de payer et restituer aux différentes parties prenantes des sommes montant à 7 ou 800000 livres $^{8}$.

Dans les deux cas, les officiers contestaient le caractère casuel de leur office, nonobstant la déclaration du mois d'août 1723, qui rétablissait la casualité des charges dans tout le royaume. Le premier, héritier de l'office de contrôleur général des domaines et bois du duché d'Orléans, auparavant détenu par un oncle qui n'avait pas acquitté le droit annuel, exerçait les fonctions de procureur du duc d'Orléans au Châtelet. Cette appartenance clientélaire favorisa un règlement à l'amiable, préféré aussi à un recours hasardeux au Conseil du roi, alors que le gouvernement était entre les mains $d^{\prime}$ '« un premier ministre [M. le duc de Bourbon] notoirement opposé aux intérêts de la maison d'Orléans » : la taxe réclamée au neveu fut annulée par une gratification d'un montant identique accordée par le duc d'Orléans9. Le contentieux qui éclata en 1746 ne pouvait être clos avec la même facilité : le plaignant, receveur général des domaines et bois du duché de Valois, avait à l'inverse indûment payé, et son père avant lui, des sommes importantes (droit annuel, droit de prêt et droits de mutation) pour un office qui n'était pas casuel mais héréditaire ${ }^{10}$ . Ce sieur Girard, de toute évidence étranger à la clientèle ducale et assuré de son bon droit, réclamait le remboursement des sommes versées, avec des intérêts. L'affaire risquait de faire jurisprudence pour toutes les créations d'offices héréditaires qui n'avaient été suivies d'aucun arrêt d'exception en faveur de l'apanage afin de conserver aux ducs le bénéfice des revenus casuels ${ }^{11}$. D'où l'estimation faramineuse de 700000 à 800000 livres à laquelle l'auteur chiffre le préjudice possible ${ }^{12}$. En effet, en présence du duc d'Orléans, son propre conseil trancha à l'unanimité moins une voix en faveur du requérant, au grand dam de notre auteur, indigné que des serviteurs aient ainsi pu manquer à leur devoir et au fondement même de leur ministère ${ }^{13}$. Cette «trahison », fruit d'un complot selon lui ${ }^{14}$, indique peut-être surtout que l'idéal ancien de la fidélité clientélaire est traversé au mitan du siècle par les valeurs contradictoires de Lumières juridiques qui transforment insensiblement les pratiques des hommes de loi. C'est retrouver, d'une certaine manière, les interrogations plus vastes formulées par Daniel Roche :

Dans la vie culturelle de la France des Lumières, à Paris comme en province, ils [les magistrats] rencontrent tous ceux qui animent le mouvement de réflexion. [...] La question posée ici est de savoir comment ces brillantes activités extra-judiciaires ont pu informer leur vie judiciaire. [...]. De Paris à la province, dans des débats laborieux, l'innovation se glisse et perdure, porteuse de rationalité et de revendications scientifiques, au cœur du XVIII ${ }^{e}$ siècle. Pas de solution de continuité entre les sphères du juridique, de l'intelligence, voire du politique, mais des interférences variables ${ }^{15}$.

Des méthodes expéditives s'imposèrent pour délivrer la maison d'Orléans de ce péril financier: le chancelier et chef du conseil ducal, conseiller d'État par ailleurs, vit son 
office changé « en une prébende de Va-t'en », pour reprendre, hors contexte, l'expression dont Charles Loyseau s'était servie afin de dénoncer l'assujettissement des juges seigneuriaux. Son successeur, le futur et éphémère contrôleur général Étienne de Silhouette, barguigna moins entre les obligations de sa charge et son attachement à la justice : il égara le jugement, une fois encore favorable aux prétentions de l'officier, que Machault d'Arnouville avait demandé à l'un des inspecteurs du domaine avant de le prévenir de n'en communiquer le résultat à personne ${ }^{16}$.

Au-delà de cette issue anecdotique, l'affaire incitait à réparer la négligence des précédents membres du conseil, d'autant qu'une kyrielle de litiges éclataient, nés du flou juridique qui entourait la nature de plusieurs offices, casuels ou héréditaires, royaux ou seigneuriaux, et les droits afférents des ducs d'Orléans ${ }^{17}$. En 1749, la destitution du lieutenant général au bailliage d'Aiguepesse par le duc d'Orléans suscite encore un procès porté en la Grand Chambre du Parlement, où le caractère seigneurial de l'office est établi, ce qui l'assimile de fait à une commission par définition amovible en dépit de l'admission de ses titulaires au paiement du prêt et du droit annuel aux parties casuelles de l'apanage ${ }^{18}$. D'autres controverses juridiques naissent quant au caractère royal ou seigneurial des offices dont les princes ont la nomination ou la pleine provision, qui mobilisent encore juristes et avocats pour la défense des privilèges singuliers des princes apanagés ${ }^{19}$.

6 En 1750, les héritiers du titulaire défunt de l'office de chevalier d'honneur au bureau des finances d'Orléans arguent de l'ignorance où il était de la nature casuelle de son office pour l'innocenter du non-paiement d'un droit annuel modique de 30 livres aux parties casuelles des Orléans. Une fois de plus, le duc fit cadeau à la veuve de la totalité de la finance qui provenait de la vente de l'office ${ }^{20}$.

7 Le discrédit dont souffre une partie des offices moyens ou subalternes de judicature, mais aussi les exigences fiscales de l'État, réitérées au milieu du siècle dans une conjoncture belliqueuse et, de surcroit, assombrie par des difficultés financières devenues structurelles, aiguillonnent les divergences entre les ducs et les officiers qui peuplent leurs domaines. Cette situation, dont les procès ne sont que la partie la plus visible, n'est pas sans rappeler les conflits multipliés dans la première moitié du $\mathrm{XVII}^{\mathrm{e}}$ siècle, consécutifs à la légalisation de la vénalitée ${ }^{21}$. Mais elle marque la fin d'une sorte d'âge intermédiaire durant lequel les officiers trouvaient un avantage indubitable à la défense vigilante des prérogatives de la maison d'Orléans dans l'apanage, les terres patrimoniales ou les domaines engagés en sa possession.

\section{L'apanage des Orléans : un monde à part de l'office moyen}

8 En effet, la croissance officière et le cortège d'expédients qui l'accompagne (dédoublements d'offices, augmentations de gages, taxes exigées pour l'attribution de droits utiles ou de l'hérédité, etc.) entrent en contradiction avec les principes juridiques qui gouvernent la jouissance des concessions domaniales des princes apanagés ou des seigneurs engagistes. En résumé, car cette question a été abordée par des travaux antérieurs ${ }^{22}$, ces innovations diminuent les revenus casuels des uns et des autres, dont les estimations, transcrites dans les lettres patentes de donation ou dans les contrats d'engagements, intègrent le produit attendu des vacances d'offices et des droits de mutation. Les résistances rencontrées au début $d u \mathrm{XVII}^{\mathrm{e}}$ siècle, non seulement à 
l'introduction de la paulette, mais aussi à celle d'offices royaux qui se surimposent et dévitalisent de facto les attributs d'officiers seigneuriaux ou domaniaux anciens, s'éclairent notamment par là. De là à affirmer que le problème du rendement économique de la possession d'offices mobilise de manière exclusive les grands seigneurs et, partant, que la perte de leur pouvoir de patronage leur serait moins sensible, il y a un pas que je ne franchirai pas. Ce serait confondre les objectifs et les modalités d'une défense contrainte de brandir, face aux exigences du pouvoir monarchique, le seul argument juridique à sa disposition, celui de l'évaluation économique inscrite dans les dispositions ou les contrats ${ }^{23}$. Au demeurant, l'affirmation liminaire du mémoire composé pour le duc d'Orléans, au cœur du xviII ${ }^{e}$ siècle, situe les enjeux sur l'échelle plus vaste de la prééminence et du prestige :

De tous les droits dont jouissent les seigneurs, il y en a peu qui puissent entrer en parallèle avec celui de pourvoir aux charges et offices et même avec celui de nommer et présenter au roi des sujets pour les remplir ${ }^{24}$.

9 Il n'en reste pas moins que la croissance officière a été ralentie, ou plutôt gauchie, dans l'apanage des ducs d'Orléans, depuis la première concession de 1626 (pour Gaston) et celle de 1661, en faveur de Philippe, qui inaugure une transmission en ligne masculine ininterrompue, et que les officiers en place ont largement bénéficié de ces dérogations à la loi commune. À mesure que la monarchie en guerre recourt aux expédients bien connus des augmentations de gages et des taxes exigibles des seuls officiers revêtus de la qualité de royaux, qui acquittent le prêt et le droit annuel aux parties casuelles du roi, la singularité des exceptions consenties en faveur de la maison d'Orléans s'est chargée d'une valeur supplémentaire, profitable aux deux parties. Plutôt qu'une récapitulation, éloquente mais fastidieuse, des dérogations obtenues durant le XVII siècle, il semble important de souligner les logiques et les justifications énoncées dans les requêtes des intéressés. Celles des ducs mettent en avant des principes invariables : tout établissement nouveau diminue le prix des offices existants, les taxes et augmentations de droits imposées ont des conséquences analogues et nuisent de surcroît au bon débit des charges, axiome qui vaut encore pour tout alourdissement des frais nécessaires aux acquéreurs, tels ceux occasionnés par la délivrance de lettres de provision royales ${ }^{25}$. Et les officiers menacés par les initiatives monarchiques se retranchent de la même façon derrière les privilèges de ceux qui les ont désignés et excipent des droits qu'ils paient exclusivement aux parties casuelles ducales. Parmi d'autres exemples, les commissaires aux saisies réelles des sièges présidiaux d'Orléans et de Chartres (longtemps sans titulaires) se pourvoient eux-mêmes au Conseil du roi lorsqu'un édit de 1689 ordonne leur suppression et leur remplacement par un officier héréditaire. Leur démarche est couronnée de succès : ils obtiennent un arrêt qui confirme leur maintien, le droit du duc à pourvoir à leurs offices demeurés casuels, et qui fait jurisprudence pour tous les titulaires de charges analogues établis dans les justices royales de l'apanage ${ }^{26}$.

Les taxes imposées aux officiers au XVII ${ }^{\mathrm{e}}$ siècle révèlent davantage le brouillage des frontières habituelles entre office royal et office seigneurial dans les domaines possédés par les Orléans. La perception du droit de joyeux avènement, il est vrai peu fréquente, indique la règle générale qui devrait être suivie pour toutes les contributions supplémentaires exigées des officiers. Elle se fonde en général sur l'origine des lettres de provision, en sorte que sont assujettis au paiement de ce droit tous ceux qui les tiennent du roi, soit la totalité du personnel des juridictions extraordinaires (bureaux des finances, élections et greniers à sel), mais aussi une partie des officiers ordinaires des sièges de 
justice royaux, pour lesquels les Orléans n'ont qu'un droit de nomination, à cause des cas royaux qui regardent la puissance publique (les présidents, les conseillers et les gens du roi des présidiaux de l'apanage ou des bailliages royaux des terres patrimoniales). Dans l'apanage, l'exemption de ce droit de confirmation s'étend aux officiers des maîtrises des Eaux et Forêts, à tous ceux des justices seigneuriales, de même qu'à l'ensemble des officiers des juridictions royales qui n'ont pas à connaître des cas royaux et pour lesquels les ducs ont donc la pleine provision et institution. À l'inverse, tous les officiers principaux des sièges de justice royaux des domaines engagés sont tenus de payer le droit de joyeux avènement, de même que ceux des terres patrimoniales. Dans ces dernières, seuls les officiers du comté de Morstain et du vicomté d'Auge, hérités des ducs de Montpensier, dont le titre de propriété se fondait sur un contrat d'échange de 1529 extrêmement avantageux, jouissent d'une exemption «totale et absolue », y compris les officiers principaux ( moyens ») des sièges de justice royaux dont les lettres de provision sont pourtant délivrées par le roi ${ }^{27}$. Le pendant approximatif de cette distinction réside dans les modalités de rétribution des officiers à gages des sièges de justice (les moyens là encore, et non les inférieurs et subalternes qui ne jouissent que de droits attribués), puisqu'ils reçoivent cette rémunération, partie du duc, partie du roi, tandis que ceux des domaines engagés sont employés dans les seuls états du roi, à l'instar de ceux des sièges extraordinaires, et que les ducs se chargent du versement de tous les gages dans leurs terres patrimoniales, où l'on considère que les sièges royaux sont maintenus « à titre de prééminence $»^{28}$.

11 Le statut insolite des officiers moyens de l'apanage reflète donc un privilège honorifique incomparable des Orléans, puisque ce droit de nomination des officiers extraordinaires, ou des officiers supérieurs des sièges de justice - que les mémoires distinguent justement des "purement seigneuriaux " comme des "purement royaux»-est octroyé depuis 1626, renouvelé en 1661, puis confirmé en 1692 pour le duc Philippe et ses successeurs mâles, et devient une partie constitutive de la concession royale alors même qu'il est retiré aux simples engagistes ${ }^{29}$. Une autre prérogative distinctive, mais utile, réside dans le versement du droit de prêt aux parties casuelles ducales ${ }^{30}$. C'est cette particularité que font valoir les greffiers en chef du bureau des finances d'Orléans, dans la requête qu'ils présentent en 1694 avec leur homologues des élections et greniers à sel de l'apanage et d'autres grands seigneurs engagistes (Condé, Vendôme, La Trémoille), afin d'être déchargés d'une taxe dont la suppression n'était acquise qu'en contrepartie d'un rachat pour les officiers qui versaient le droit de prêt aux coffres du roi ${ }^{31}$. Ces subtilités, marques de la créativité fiscale dont l'État en guerre a fait preuve pour réduire les enclaves du privilège, expliquent pourquoi l'argument juridico-économique des revenus de l'apanage demeure, en dernier ressort, le plus efficient. Il légitime la dérogation à l'application des édits particuliers ou généraux attributifs de l'hérédité. Ainsi, la situation exceptionnelle des officiers royaux désignés par les Orléans n'est sans doute jamais aussi patente qu'après l'édit de décembre 1709, qui impose à tous ceux astreints au paiement de l'annuel et du prêt le rachat de ces droits en contrepartie de la concession de l'hérédité, et en raison duquel Jean Nagle soulignait combien la défaite de Malplaquet avait été plus cruelle à l'office que la Fronde ${ }^{32}$. Cette singularité persista une bonne douzaine d'années, pendant lesquelles tous les officiers royaux des domaines des Orléans continuèrent d'alimenter les parties casuelles ducales, jusqu'au rétablissement général de la casualité des offices, effectif dès 1723 :

On s'est toujours conformé dans l'apanage aux mêmes règlements des parties casuelles du roi, à l'exception seulement du rachat du prêt et du droit annuel qui 
fut ordonné en 1709 dans tout le royaume. L'apanage en fut exempté et feu S.A.R. ne jugea pas qu'il fut de l'intérêt de ses parties casuelles de demander le rachat dudit prêt et annuel, tant par rapport aux différents effets royaux qui étaient pris en paiement dudit rachat, que pour d'autres bonnes raisons qui conviennent au bien de son apanage, ainsi le prêt et le droit annuel continua d'être payé par tous les officiers de son apanage, terres patrimoniales et domaines tenus par engagement dont les offices étaient anciennement casuels ${ }^{33}$.

Il serait intéressant de vérifier si les multiples aléas, les taxes épargnées à ces officiers royaux prompts à exploiter et à faire valoir les prérogatives de ceux qui les ont pourvus, ont eu un impact dans les contrats de vente entre particuliers, si l'entrée dans ce monde à part de l'office moyen et inférieur fut davantage recherchée au XVII siècle. À tout le moins, cette logique axée sur le privilège paraît moins adaptée à la conjoncture fiscale et à la vitalité défaillante du marché d'une partie des offices moyens au siècle suivant.

\section{L'érosion de la rhétorique du privilège}

13 S'il est vrai que l'argumentation juridico-économique avait déjà joué dans un sens unique, lors des créations d'offices effectives, compensées par des indemnités ou la promesse de revenus équivalents ou supérieurs à la perte que les Orléans étaient supposés souffrir, elle forme dans la première moitié du XVIII ${ }^{\mathrm{e}}$ siècle un obstacle à l'application des dispositions mises en œuvre pour remédier à la désaffection qui frappe l'office moyen de judicature. Ainsi, la réduction de moitié des droits de prêt et annuel, après 1723, n'a pas lieu dans l'apanage dont elle diminuerait les revenus casuels $^{34}$. Ces derniers sont pourtant gravement affectés par le discrédit des offices moyens et inférieurs de justice, si l'on en croit les analyses du Mémoire pour servir d'instruction aux parties casuelles de Mgr le duc d'Orléans:

Presque toutes les évaluations tant anciennes que nouvelles ont été tirées du registre des évaluations des offices de France aux parties casuelles du roi, c'est ce qui a empêché le conseil de S.A.S. de les réduire à une moindre somme, quoiqu'il y ait beaucoup d'offices principalement dans la magistrature, qui sont absolument trop évalués, ce qui empêche les officiers de payer le prêt et le droit annuel. Ils aiment mieux laisser tomber leurs charges vacantes par leur mort aux parties casuelles de S.A.S. où elles restent quelquefois longtemps à lever. Par exemple, les offices de conseillers aux présidiaux d'Orléans et de Chartres qui sont évalués à 5333 livres 6 sols 8 deniers sont tellement tombés que l'on n'en trouve pas 3000 livres quand ils sont vacants. Il est vrai qu'autrefois ces offices valaient 5 à 6000 livres, mais on n'en trouvera plus à ce prix et les choses ont bien changé de face. Les autres évaluations des offices de judicature sont aussi trop fortes et excepté les chefs qui ont obtenu des modérations il y en a peu qui paient le prêt et le droit annuel. Tous les petits offices de procureurs, notaires, huissiers et sergents sont aussi trop fortement évalués de sorte que pour remettre le prêt et le droit annuel en valeur, il faudrait, pour ainsi dire, refondre toutes les évaluations par rapport au temps présent et c'est à quoi le conseil n'a pas voulu toucher ${ }^{35}$.

Ces remarques témoignent du dilemme né de difficultés jusqu'alors ignorées, mais peutêtre jugées passagères, ce qui expliquerait la recherche de solutions qui n'exposent pas à un effondrement durable des revenus casuels ducaux, risque encouru avec le choix, rejeté, d'une refonte des évaluations utilisées pour la fixation des droits. De fait, le conseil princier lui préfère des remèdes temporaires. Il tempère ainsi ses exigences à l'égard des officiers royaux du bureau des finances d'Orléans, dont il avait d'abord exploité, à la suite du pouvoir royal, le privilège d'être exemptés du droit de prêt : 
Ceux [les officiers du bureau des finances] d'Orléans, qui payaient autrefois en corps une finance assez considérable à feu Monsieur et à feu S.A.R. à chaque renouvellement de prêt pour être exemptés dudit prêt, ont été traités plus favorablement depuis les trois derniers baux. Le Conseil de S.A.S. a fait un abonnement avec la plus forte partie des officiers dudit bureau qui se sont conservés et se sont obligés de payer solidairement et en nom collectif une somme de 360 livres chacun par chacun an tant pour le prêt que pour le droit annuel. Ce n'est qu'une augmentation de 26 livres 13 sols 4 deniers chacun par an, le droit annuel des trésoriers de France étant fixé par chacun an à 333 livres 6 sols 8 deniers, chacun suivant l'ancienne évaluation. Ainsi ces messieurs sont pour ainsi dire exemptés du prêt vu la légère augmentation, mais ceux du corps qui n'ont pas voulu entrer dans la confédération et abonnement sont traités à la rigueur et leur décès arrivant leurs charges tombent vacantes au profit de S.A.S. comme il est arrivé depuis peu à celle du sieur Bagnault de Montault ${ }^{36}$.

Le rejet ou l'incapacité de quelques individus à satisfaire au paiement de cette taxe à l'exemption de taxe, même modérée et parée des couleurs de la grâce ducale, sont une réalité assez forte pour briser dans le cas présent la solidarité des corps d'officiers sur laquelle la monarchie absolue a fondé, au moins depuis le règne de Louis XIV, sa dialectique fiscale nourrie de la «relation symbiotique» qu'elle entretenait avec le privilège ${ }^{37}$.

Et le Mémoire dévoile d'autres symptômes d'un affaiblissement de la puissance persuasive de la rhétorique du privilège, face à la détermination fiscale nouvelle de l'État au milieu $\mathrm{du} \mathrm{XVIII}^{\mathrm{e}}$ siècle. D'abord, les réflexions du rédacteur du traité, qui regarde rétrospectivement la majeure partie $\mathrm{du} \mathrm{XVII}^{\mathrm{e}}$ siècle comme un apogée des prérogatives de l'apanage et de ses possesseurs, ne peut manquer de frapper tout historien dixseptiémiste, accoutumé à scruter une offensive multiforme du pouvoir monarchique commencée avec Richelieu et parachevée pendant le règne personnel du Roi-Soleil ${ }^{38}$. Certes, ce texte procède largement d'une relecture historique qui répond à une démarche, de plus en plus usitée au XVIII ${ }^{\mathrm{e}}$ siècle, de justification par un passé investi d'une valeur probatoire. Il reproduit et adapte à sa chronique des offices et de l'apanage une vision tripartite du temps et de l'évolution très répandue chez les contemporains ${ }^{39}$. Sa seconde période, celle des privilèges sauvegardés et des transactions acceptées en raison des nécessités fiscales accrues du pouvoir royal belliqueux de Louis XIV, débute dans les années 1690 :

$\mathrm{Au}$ reste, sous le règne de Louis XIII il n'a été créé qu'un petit nombre de nouveaux offices, soit de judicature, ou de finance; Il n'en a pas été de même sous le règne suivant, qui est celui où l'apanage a été concédé à S.A.R Monsieur Philippe [...]. Sous le roi Louis XIV, environ 45 ans après son avènement à la couronne (vers 1690), la création de nouveaux offices est devenue très fréquente, et pour ainsi dire, journalière. [...] il est à présumer que les motifs des édits de création (et par là on entend les besoins de l'État) ont été ceux du prince (S.A.R. Monsieur Philippe $\mathrm{I}^{\mathrm{er}} \mathrm{du}$ nom) par qui l'apanage était alors possédé, pour consentir à ce que, dans son étendue, il soit établi de nouveaux offices, mais sous la condition qu'après le paiement fait au roi de leur première finance, ils demeureraient casuels, et comme tels sujets aux parties casuelles de l'apanage ${ }^{40}$.

Le troisième temps, celui du privilège bafoué, tant dans l'apanage que dans les terres patrimoniales, est inauguré dans les années 1740, lorsque la monarchie impose des taxes sur les offices et devient sourde aux arguments juridico-économiques habituels, qu'elle récuse dans ses réponses et lèse par sa pratique. De multiples articles du traité portent témoignage de ce durcissement qui n'épargne plus les moyens et petits officiers de l'apanage et des terres patrimoniales des Orléans, désormais employés dans les rôles de 
taxes. Par exemple, tous ceux des Eaux et Forêts le sont pour le rachat de l'annuel et du droit de prêt (qu'ils ne payaient pourtant pas au roi) et l'attribution de droits utiles, conformément aux dispositions d'un édit de février $1745^{41}$. De même, les receveurs des consignations et commissaires aux saisies réelles se voient-ils acculés à financer des augmentations de gages en conséquence d'un édit de décembre 1743, lorsque Machault d'Arnouville confirme en 1747 qu'il ne tolère aucune dérogation ${ }^{42}$.

Enfin, les officiers des élections et greniers à sel de l'apanage, s'ils sont déchargés du rachat du prêt et de l'annuel imposé par l'édit de février 1745, n'en doivent pas moins financer pour acquérir les droits qui leur sont attribués, de deniers pour livre du montant du rôle des tailles pour les premiers, et de cinq sols des droits imposés sur chaque minot de sel pour les seconds. Le Mémoire entérine le coup de force, se contentant de souligner que cette finance forme pour eux un "genre de bien totalement distinct et séparé du corps de l'office ", qui passe aux héritiers en toutes circonstances, même en cas de nonpaiement du droit annuel ou de ceux de mutation aux ducs ${ }^{43}$.

18 Loin de continuer à réclamer une indemnité du roi, souvent accordée mais de moins en moins effectivement versée, au titre de la compensation des pertes infligées à ses parties casuelles, le duc d'Orléans se trouve souvent contraint de payer en personne les finances exigées, au premier chef pour les offices domaniaux dont il est propriétaire et qui sont exercés par des titulaires ou des commis ${ }^{44}$. Il dépense ainsi près de 135000 livres en 1749 au titre des taxes imposées aux officiers des Eaux et Forêts, à condition toutefois de jouir d'une partie des droits utiles qui leur sont attribués. Si le traité considère ces versements comme le fruit que de la "pure bonté » du duc, conscient que «ses officiers étaient hors d'état d'y satisfaire $»^{45}$, cette générosité gratuite est démentie par les circonstances dans lesquelles le prince apanagé acquiert des offices municipaux. Il avait déjà pris une initiative de cette nature en 1730, offrant 200000 livres pour ceux de receveurs des octrois, rétablis en titre d'office, avant de traiter séparément avec les villes et communautés désireuses de les réunir à prix d'argent afin de conserver leur liberté de désignation ${ }^{46}$. Mais il fait montre d'une véritable boulimie d'achats après le rétablissement des offices municipaux réglé par les édits de 1733 et 1734 : il se rend propriétaire, moyennant 30000 livres et le droit de 6 deniers pour livre, des deux offices de maire et de procureur du roi de la ville de Chaumont-en-Bassigny, qui fait partie de ses domaines engagés de même que celle de Honfleur où il achète aussi les offices de maires en 1746.

Enfin, il procède à des acquisitions ponctuelles dans les villes qui dépendent de ses terres patrimoniales, telle Joinville, pour sept offices d'échevin en 1746, puis à Villefranche-enBeaujolais, en 1750, avec deux autres offices d'échevin ${ }^{47}$. Si le Mémoire allègue en l'occurrence le souci ducal du «repos et de la tranquillité de certaines villes", il mentionne aussi les difficultés nées pour le remboursement des titulaires, forts de l'édit de création qui spécifiait que les acquéreurs ne pouvaient être dépossédés pour quelque cause que ce soit. Il fallut attendre la mort des détenteurs ou les convaincre de rétrocéder leur office, et c'est le motif véritable de ces achats échelonnés.

Cette fièvre acquéreuse résulte aussi de l'autoritarisme fiscal de la monarchie. Confronté à la mévente des offices en question, le Conseil autorisa en effet leur réunion aux corps de ville et établit de nouveaux droits fiscaux dont la jouissance leur serait concédée jusqu'au parfait paiement de la somme. Or quelques corps de villes, notamment dans l'apanage, avaient devancé le duc et fait des offres au Conseil pour racheter ces offices: celui d'Orléans ne débourse pas moins de 180000 livres outre les 10 deniers pour livre, tandis 
que ceux de Chartres et de Beaugency, aussi inclus dans l'apanage, versent respectivement 9000 et 10000 livres à cette fin ${ }^{48}$.

21 Le duc avait donné son agrément, à condition d'être maintenu dans son droit de nomination et en contrepartie du versement par les villes d'une indemnité annuelle de 400 livres pour Orléans, 200 livres pour Chartres et de 30 livres pour Beaugency, en vertu du principe habituel de la compensation. Cependant, ces créations et surtout les réunions d'offices menaçaient de porter une atteinte durable aux revenus ducaux, d'autant que l'édit de 1734 attribuait exclusivement les droits d'annuel ou de mutation de ces offices aux parties casuelles du roi. C'est la raison pour laquelle le duc d'Orléans réclama et obtint, en mai 1749, un arrêt du Conseil qui confirmait son droit de percevoir le droit annuel et les profits des mutations des offices réunis. L'arrêt faisait encore obligation aux communautés d'élire et de lui nommer un nouveau sujet dès la mort du premier désigné. Toutefois, les critères de choix des individus choisis trahissent des logiques économiques désormais divergentes, sinon antagoniques, des ducs et des possesseurs d'offices, collectifs ou non. Car les assemblées électorales réunies dans les villes acquéreuses des offices et des droits optent de manière générale pour les sujets « dont la santé paraît la plus ferme afin de reculer autant que possible le paiement des frais des nouvelles lettres de grand sceau ${ }^{49}$ qui devait tenir lieu de lettres de provision.

Ces stratégies désormais séparées, à travers lesquelles le comportement des corps de ville situés dans les domaines des Orléans s'aligne maintenant sur celui des autres villes du royaume, sont un indice supplémentaire de la dislocation du front, jusqu'ici uni, des officiers et des ducs intéressés à la défense des droits particuliers de l'apanage. C'est l'une des conséquences remarquables de la perturbation introduite par l'agressivité fiscale montante du pouvoir dans l'enclave jusqu'ici préservée du monde de l'office moyen des ducs d'Orléans. Les sommes déboursées par le duc, après les échecs répétés de ses représentations au Conseil, les exigences fiscales qui frappent désormais les officiers, confirment la chronologie de l'entrée dans une nouvelle phase de l'absolutisme, établie par David Bien et confirmée dans le cas des taxations universelles par le récent ouvrage de Michaël Kwass, qui consigne l'avènement, au milieu du XVIII ${ }^{\mathrm{e}}$ siècle, d'un nouveau type de sujet, le "contribuable privilégié payeur de $\operatorname{taxes}^{50}$ ", catégorie dans laquelle se rangent désormais les premiers princes du sang eux-mêmes et leurs officiers royaux, moyens et subalternes.

Alors que le rempart du privilège, pleinement opérant ou à peine entamé sous les règnes de Louis XIII et de Louis XIV, se fissure sous les coups de boutoir fiscaux répétés du pouvoir royal, l'atonie du marché des offices moyens se trouve peut-être aggravée, dans l'apanage des Orléans, par le principe économique qui régit celui-ci et détourne des diminutions consenties par la monarchie elle-même (il faudrait, pour s'en assurer, analyser des contrats de vente entre particuliers, car les registres des parties casuelles ont presque tous disparu). Enfin, signe ultime de la prise de conscience de l'érosion de la force persuasive de la rhétorique du privilège, les mémoires composés au nom du duc d'Orléans pour la décharge des officiers comptables, sans renoncer au discours habituel d'une justice attendue du roi, lui adjoignent soudain des arguments puisés dans le registre nouveau de la pensée politique, auxquels le traité attribue, du reste à tort, un potentiel de conviction supplémentaire :

Parmi les moyens rassemblés dans ces mémoires, le principal était que les officiers

dont il s'agit ici sont à la vérité comptables, mais envers le public seulement, sous

l'autorité des magistrats, et non pas envers S. M.51. 
Aussi ces mémoires multiples forment-ils davantage qu'un constat navré mais impuissant de la décadence des prérogatives des princes apanagés, le matériau de fabrication de ripostes juridiques et historiques ou pseudo-historiques encore tâtonnantes. Elles étaieront, dans la seconde moitié du siècle, une défense efficace des prérogatives et des revenus casuels d'un apanage agrandi, consolidé par l'intégration d'une fraction croissante des domaines engagés et même des terres patrimoniales des princes (lors de sa suppression, il fournit près des deux tiers des revenus annuels de Philippe Égalitéf ${ }^{52}$ ), mais devenu l'objet de controverses inédites quant à la nature de la possession et à l'étendue des pouvoirs de ses détenteurs ${ }^{53}$.

\section{NOTES}

1. David Bien, «Les offices, les corps et le crédit d'État : l'utilisation des privilèges sous l'Ancien Régime ", Annales ESC, mars-avril 1988, p. 379-404, p. 382.

2. «Officiers «moyens" (I), Cahiers du Centre de recherches historiques, $\mathrm{n}^{\circ} 23$, octobre 1999 , en particulier Michel Cassan, «L'activité du présidial de Limoges (fin XVII ${ }^{\mathrm{e}}$ siècle-fin $\mathrm{XVIII}^{\mathrm{e}}$ siècle), p. 29-47, et Christophe Blanquie, «Les épices du lieutenant général de Libourne (1725-1755), op. cit., p. 61-72.

3. Ainsi, dans les domaines possédés par la maison d'Orléans, la prévôté de Beaugency est réunie au bailliage de la ville en 1738, de même que celle de Compiègne l'est en 1748 ; celle d'Orléans disparaît en 1749, au moment où un nouveau bailliage voit le jour à Honfleur, et qu'un autre est déplacé dans la ville de Dompfront, en 1753. En 1755, un nouvel édit distrait la prévôté de Pierrefonds du bailliage de Compiègne pour la réunir au siège présidial de Soissons.

4. Arch. nat., K 652 (4), fo 139

5. Arch. nat., $\mathrm{K} 652(3,4)$, Traité des droits et prérogatives de Monseigneur le duc d'Orléans relativement aux juridictions, charges, offices et places qui dépendent de ses duchés, seigneuries et terres possédées à titre d'apanage, patrimonial, d'engagement du roi divisé en deux parties et rédigé par ordre alphabétique, 1747 (première partie), 1750 (seconde partie).

6. Arch. nat., $\mathrm{K} 652,(3), \mathrm{f}^{\circ} 2$, préface.

7. Arch. nat., K 652, (4), $\mathrm{f}^{\circ} 76$.

8. Ibid., fo 73 .

9. Arch. nat., K 652, (4), fo $76:$ : [...] le neveu avait en sa faveur et l'importance de ses fonctions dans sa charge et l'avantage de représenter un oncle qui pendant plus de trente années avait rendu à la maison des services très essentiels ".

10. C'est en décembre 1689 et en mai 1702 qu'ont été créés des offices héréditaires de receveurs généraux des bois et domaines, ancien et alternatif.

11. La recherche de textes juridiques pour cette affaire découvre qu'une dizaine d'édits portant création d'offices héréditaires, entre 1686 et 1702, n'ont pas été accompagnés de règlements spéciaux pour ceux possédés par la maison d'Orléans.

12. Arch. nat, $\mathrm{K} 652$ (4), fo 75 : « Il ne faut cependant pas dissimuler que, si dans 25 occasions, ou environ, de création de nouveaux offices, avec attribution de l'hérédité, ceux établis dans l'apanage ont été déclarés casuels, au profit du prince, il y a d'un autre côté quelquefois été établi des offices ayant la même attribution, sans qu'il ait été rendu des règlements à l'effet de la retrancher. Peut-être le conseil de la maison a-t-il négligé de faire, dans ces circonstances, les 
démarches pour l'obtention d'une exception, à cause de la sécurité que paraissait inspirer la multiplicité de lettres accordées dans le même cas ». L'intendant, comme le précise un autre passage du texte, était M. Héricart de Tury, conseiller en la cour des aides de Paris.

13. Arch. nat., K 652, (4), fo 77.

14. "Le sieur Girard ne rencontra qu'un seul contradicteur en la personne du même officier dont le chef avait soigneusement évité le ministère. Ainsi le prince se trouvait (ose-t-on se servir du terme) condamné par l'avis presque unanime de son Conseil à la restitution de 7 ou 800000 livres, dans lesquelles le sieur Girard avait à espérer le recouvrement de sommes d'autant plus considérables que, par son mémoire, il avait conclu en des intérêts à compter du jour des paiements fait, tant par lui que par son défunt père. Jusque là tout répondait à son espérance. Rapporteur choisi avec une sorte de préméditation, son opinion entraînant, pour mieux dire subjuguant les suffrages de tous les assistants, à l'exception d'un seul. Enfin chacun des deux protecteurs de son système [le chancelier et l'intendant rapporteur de l'affaire] porte l'attention au point de lui épargner, par le moyen de lettres. On trouvera dans le cahier relatif à cette affaire les copies de ces deux lettres, et le lecteur est invité à y jeter les yeux. Dans la première en date, savoir celle de l'intendant, on a peine à reconnaître et la conduite et le langage dont était tenu un officier qui, dès lors, comptait plus de trente années d'exercice dans une charge de judicature en cour supérieure, et le détail qu'elle contient est bien contraire à la teneur des deux serments qu'il avait prêtés en entrant soit dans sa juridiction, ou dans le conseil de la maison [...] l'inquiétude où il pouvait être touchant la décision et ses circonstances ", ibid., fo 77.

15. Daniel Roche, La France des Lumières, Paris, Fayard, 1993, 651 p., p. 279-280.

16. Arch. nat., K 652 (4), fos $78 \mathrm{v}^{\mathrm{o}}-79$. Ce « dire » de l'inspecteur du domaine s'appuyait pourtant sur un nouveau mémoire composé à la demande de Silhouette, favorable aux intérêts du prince et qui rallia cette fois les suffrages de son conseil. Et les premières délibérations du conseil princier, favorable à Girard, ne furent pas transcrites par écrit : « sans entreprendre de pénétrer les causes de cette omission, on se borne à la regarder comme en quelque façon heureuse pour le prince, à cause des difficultés qu'aurait pu occasionner la réformation d'une espèce de délibération revêtue de sa forme ordinaire ", fos $77 \mathrm{v}^{\mathrm{o}}-78$.

17. «Par leur nature, tous les offices vénaux sont casuels et périssables, faute du paiement du prest et de l'annuel. Mais (surtout vers la fin du dernier siècle) il a plu au roi d'attribuer l'hérédité à différents officiers, et ainsi rien de plus important, pour l'objet proposé, que de connaître avec certitude quel a été à l'égard des terres et seigneries que possède $\mathrm{M}$. le duc l'effet des édits portant (en faveur de certains offices) attribution de l'hérédité ", ibid., fo 2.

18. Arch. nat., $\mathrm{K} 652$ (3), fo $100 \mathrm{v}^{\mathrm{o}}-101$, Ce sont les termes rapportés de la plaidoirie de l'avocat général du prince.

19. Arch. nat., K 557 (1). Mémoire pour servir d'instruction aux parties casuelles de Mgr le duc d'Orléans. 20. Arch. nat., K 652, (3), fo 122.

21. Roland Mousnier, La Vénalité des offices sous Henri IV et Louis XIII, $2^{\mathrm{e}}$ édition, Paris, PUF, 1971, notamment p. 322-324, où sont mentionnés des abus divers des possesseurs de parties du domaine aliéné, engagistes ou apanagés (vente des offices à un prix excessif, taxes très élevées des résignations, attente de la mort de l'officier malgré la dispense des 40 jours permise par le paiement de la paulette, afin d'augmenter les vacances d'offices et les revenus de leurs parties casuelles), pratiques dénoncées par les députés du tiers état en 1615 ou les états de Normandie en 1624.

22. Roland Mousnier, op. cit.., p. 313-325.

23. «[...1 Le Roy, en différents temps, a créé de nouveaux offices, mais tous les édits à cet effet rendus n'ont pas eu indistinctement leur exécution, dans l'étendue de toutes les terres et seigneuries de Mgr le duc d'Orléans sur le fondement des privilèges attachés à certaines de ces terres, le Roi, dans plusieurs occasions, a jugé conforme à sa justice de les excepter de l'établissement d'offices nouvellement créés ", arch. nat., K 652, (4), fo 2. 
24. Arch. nat., K 652, (4), fo $2 \mathrm{r}^{\circ}$.

25. Ibid., fos $27,31,117,145$.

26. Ibid., (3), p. 48-50.

27. Arch. nat., K 652, (4), fo 10-14.

28. Ibid., fos 53 et 69 .

29. Arch. nat., K 652, (3), fo 109 bis. Quelques grands engagistes avaient cependant obtenu des dérogations, mais personnelles et viagères, comme la Grande Mademoiselle, qui ne transmit pas ce privilège à ses héritiers pour le domaine engagé de Dompfront.

30. Ibid., (3), fo $44 \mathrm{r}^{\circ}$. Le texte explique que ce droit a succédé au droit royal établi en 1642, lequel ne pouvait jamais s'étendre aux offices dont la pleine provision appartenait au prince apanagiste. Tous les officiers royaux qui payaient le droit annuel à Gaston d'Orléans avaient été déchargés des taxes demandées pour l'extinction de ce droit en 1646.

31. Arch. nat., $\mathrm{K} 652$, (4), fos $63 \mathrm{v}^{\circ}-64$.

32. Marcel Pinet (dir.), Histoire de la fonction publique en France, 3 vol., Paris, Nouvelle Librairie de France, 1993, t. II, « Du XVI ${ }^{\mathrm{e}}$ au XVIII ${ }^{\mathrm{e}}$ siècle », p. 196.

33. Arch. nat., K 652, fo 69 ; K 557 (1), nf.

34. «Les parties casuelles du roi ont si bien reconnu la décadence des présidiaux que Sa Majesté a ordonné que les officiers seraient reçus au paiement du prêt et droit annuel sur le pied de la moitié des évaluations d'iceux depuis le rétablissement du prêt et annuel arrivé en 1723, ce qui n'a point été suivi dans l'apanage où les choses sont toujours restées sur l'ancien pied. ", arch. nat., K 557, (1), nf.

35. Ibid.

36. arch. nat., K 557, (1), nf.

37. David Bien, art. cit., p. 381 et 397.

38. «Pendant la possession de Monsieur Gaston, son apanage a été excepté non seulement de taxes imposées, mais encore de la création de nouveaux offices. [...] et il semble permis d'avancer, qu'alors pour se soutenir les privilèges de l'apanage se suffisaient à eux-mêmes (tout le monde sait que nonobstant la qualité de présomptif héritier de la couronne, Monsieur Gaston s'est absenté plusieurs fois de la cour, et qu'il est une fois sorti hors du royaume. ", arch. nat., K 652, (4), $\mathrm{f}^{\circ} 172$.

39. Michaël Kwass, Privilege and the Politics of Taxation in Eighteenth-Century France, Cambridge, Cambridge University Press, 2000, 353 p., p. 162.

40. Arch. nat., K 652, (4), folos $173-174$.

41. Ibid., fos $100-102$.

42. Ibid., fos 137-138.

43. Arch. nat., $\mathrm{K} 652,(4)$, fos $46 \mathrm{v}^{0}$ et 135.

44. Les notaires, procureurs, huissiers des juridictions royales des domaines engagés des princes sont aussi contraints de financer l'attribution de l'hérédité aux termes d'un véritable chantage fiscal commencé en 1743 et parachevé en 1750 par une déclaration et un arrêt du conseil qui suppriment les offices de tous ceux qui n'ont pas acquitté ce droit et interdisent leurs titulaires de toutes leurs fonctions à peine d'amende, ibid., fos $70 \mathrm{v}^{\mathrm{o}}-73$.

45. Ibid., (4), fo 176.

46. Ibid., (3), fos 164-166.

47. Arch. nat., K 652, (4), (4), fos 129-132.

48. Ibid., fos $129-130$.

49. Ibid., fo 135 .

50. David Bien, art. cit., p. 389-390 ; Michaël Kwass, op. cit., p. 114.

51. Arch. nat., K 652, (4), fo 138.

52. Si l'on inclut le produit des maisons louées et vendues autour du Palais-Royal (qui fait partie de l'apanage), les revenus des biens apanages représente près de $65 \%$ d'un revenu annuel estimé 
à plus de 8,9 millions de livres en 1790. Je tiens à remercier chaleureusement Olivier Dautresme, qui achève une thèse sur le Palais-Royal à la fin $\mathrm{du}$ XVIII ${ }^{\mathrm{e}}$ siècle, de m'avoir communiqué les documents grâce auxquels j'ai pu me livrer à ce calcul.

53. Béatrice Hyslop, L'Apanage de Philippe Égalité, duc d'Orléans (1785-1791), Société des études robespierristes, Paris, 1965, 453 p., p. 11-15. Les avocats des prérogatives royales, et en particulier Du Vaucel, se voient précisément opposer l'édit de 1751 et l'arrêt de 1766, qui justifient les réunions de terres engagées à l'apanage comme un moyen de préparer le retour au domaine royal de terres qui en avaient été distraites, et qui seraient par là de l'intérêt commun des ducs et de la Couronne. 